\title{
Estudio de la agricultura urbana y periurbana de Varzea Grande (MT) bajo la perspectiva del análisis de clusters
}

\begin{abstract}
La provincia de Mato Grosso es conocida globalmente por su amplia producción agropecuaria, altamente tecnificada y con contributo expresivo a la seguridad alimentaria. Sin embargo, se identifica una característica infrecuente en el municipio de Varzea Grande, junto a capital Cuiabá, de experiencias con agricultura urbana y periurbana, que perduran durante años y cumplen un papel representativo para con la producción de alimentos y combate a problemas de salud pública locales. Por ser considerada una actividad importante del punto de vista socioeconómico y ambiental, se objetiva con este estudio analizar a referida estructura de producción, de característica horticola, su importancia, magnitud, limitaciones y potencialidades para el referido municipio y entorno. En el primer momento se hace una breve contextualización de la actividad, por medio de un estudio exploratorio, de carácter empírico y bibliográfico, con destaque para los aspectos socioeconómicos y, en la secuencia, se realiza un Análisis de Agrupaciones (Clusters) con base nos datos primarios colectados junto a los productores locales. A pesar de la parcela de alimentos producidos y manutención de los terrenos libres de insectos o animales transmisores de enfermedades, los resultados apuntan para una actividad en vías de degradación, cuya la rentabilidad y proceso de comercialización a tornan poco atractiva o complementaría de renta. El cuadro general de la actividad en el municipio engloba pequeñas fuerzas productivas que no consiguen sostenerse en la actual estructura de mercado, o mismo a la acción prolongada de una política social desfavorable, motivos que intensifican su proceso de deterioro.
\end{abstract}

Palavras-chave: Agricultura Urbana; Horticultura; Análisis de Agrupaciones; Varzea Grande.

\section{Urban and periurban agriculture study on Varzea Grande (MT) under the perspective of cluster analysis}

\begin{abstract}
The Mato Grosso province is known globally for its wide agricultural production, high technology and significant contribution to food security. However, it identifies an unusual feature in the city of Varzea Grande, near the capital Cuiaba, experience with urban agriculture that last for years and play a representative role towards food production and combat public health problems local. As it is considered an important activity of the socioeconomic and environmental point of view, the objective of this study is to examine this production structure of the vegetable crop, their importance, magnitude, limitations and potential for the municipality and surrounding areas. At first we make a brief background of the activity, through an exploratory study of empirical and bibliographical, highlighting the socio-economic aspects and, as a result, held a cluster analysis based on the collected primary data together local producers. Although the share of food produced and maintenance of vacant land of insects or disease vectors animals, the results point to an activity in degradation pathways, whose profitability and marketing process make it unattractive or complement income. The overall picture of activity in the municipality includes small productive forces that cannot be sustained in the current market structure, or even prolonged action of an unfavourable social policy reasons that intensify its deterioration process.
\end{abstract}

Keywords: Urban Agriculture, Horticulture, Cluster Analysis, Varzea Grande.

\section{Topic: Valoração e Economia Ambiental}

Reviewed anonymously in the process of blind peer

Diogo Jose Amorim de Almeida

Universidade Federal de Mato Grosso, Brasi

http://lattes.cnpq.br/8994087583791569

diogo07almeida@gmail.com

Dilamar Dallemole

Universidade Federal de Mato Grosso, Brasil

http://lattes.cnpq.br/2556871852182132

dilamar@ufmt.br

Arturo Alejandro Zavala Zavala

Universidade Federal de Mato Grosso

http://lattes.cnpq.br/0725525983034054

arturoz@ufmt.br

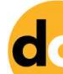

DOI: 10.6008/SPC2179-6858.2016.002.0019
Received: 15/02/2016

Approved: 20/04/2016
Referencing this:

ALMEIDA, D. J. A.; DALLEMOLE, D.; ZAVALA, A. A. Z.. Urban and periurban agriculture study on Varzea Grande (MT) under the perspective of cluster analysis. Revista Ibero-Americana de Ciências Ambientais, v.7, n.2, p.225-241, 2016. DOI: http://doi.org/10.6008/SPC2179-6858.2016.002.0019 


\section{INTRODUÇÃO}

La provincia de Mato Grosso es conocida por su actividad económica agroexportadora, sin embargo esta realidad no se presenta en todos los municipios. Algunos de estós poseen sus principales actividades económicas sostenidas en los demás sectores productivos, como es el caso de Varzea Grande, cuyas principales actividades estaban relacionadas a la confección de artefactos y a plantaciones agrícolas de subsistencia. Fundada por José Vieira Couto Magalhães (Presidente Provincial) el 15 de mayo de 1867, se volvió municipio el 23 de septiembre de 1948, en el gobierno de Arnaldo de Figueiredo, seis años despues la construcción del primer puente que liga la actual capital Cuiabá, aumentando significativamente el comercio e interacción entre los municipios.

Otrora conocida como ciudad industrial, Varzea Grande tiene en su histórico la inmigración y la cesión de áreas públicas como factor de incentivo la instalación de industrias, sin embargo, el nombre ya no refleja el principal sector económico en el municipio. Según datos del Instituto Brasileño de Geografía y Estadística (IBGE), en el año de 2010, el nicho económico principal estaba relacionado al sector de servicios, responsable por el $76 \%$ del PIB municipal, seguido por el sector industrial, que representaba el $22,8 \%$ del total y por el sector primario cuya parcela de participación fuera de apenas el 1,2\%.

Los números presentados están relacionados a una característica común por todo estado: la dualidad en la agricultura, o sea, la presencia de la agricultura altamente tecnológica y rentable en algunas regiones y la agricultura más rústica, generalmente familiar en otras. De acuerdo con Silva (2010), en Varzea Grande predomina la agricultura rústica, localizada generalmente en las tres demarcaciones rurales del municipio. A pesar de presentar inexpresiva importancia de modo general, la actividad económica municipal es de conocimiento tácito que muchas familias en la zona rural del municipio logran renta por medio de la actividad.

Al percibir el potencial de la actividad como forma de generación de renta, la Secretaría de Medio Ambiente y Agricultura (SEMA/VG), en el año de 2012, efectuó acciones de auxilio la agricultura que variaban desde la enseñanza de técnicas de irrigación y preparación de la tierra, hasta la donación de semillas y calcáreo, sin embargo, tales acciones se restringían la agricultura familiar en las demarcaciones rurales. Tal limitación se vuelve preocupante desde el momento que se observan experiencias de agricultura urbana que perduran durante años en el municipio sin cualquier incentivo, específicamente, experiencias de Horticultura.

La preocupación con la actividad citada, a pesar de inexpresiva participación en el PIB municipal, gana mayor consistencia cuando considerado el contexto político-administrativo en el cual el municipio se encuentra. En la última década, Varzea Grande presentó una serie de irregularidades administrativas, que están relacionadas con la seguridad alimentaria y la salud pública. Desde 2010 las escuelas y las creches municipales encuentran problemas con el suministro de comidas escolares, a punto de suspender las clases, una vez que la mayor parte de los alumnos de la red municipal de enseñanza es oriunda de familias carentes y encuentran en la comida escolar la principal forma de alimentación. La situación culminó en la instauración 
un expediente civil público en 2011 para apurar las irregularidades en la comida escolar en el municipio, sin embargo, en 2012 el suministro continuó irregular, de modo que hubo casos en los que la propia dirección de la escuela abriera cuenta en establecimientos comerciales y, con el desembolso colectivo de padres y maestros, adquirió los alimentos.

Al dirigir el horizonte para la cuestión de la salud pública, se apunta el problema del surto de dengue que, allende Varzea Grande, registrase números preocupantes en la vecina capital Cuiabá, Rondonópolis y Sinop. En el año de 2012 fueron registrados 7.275 casos de dengue en Mato Grosso, siendo 890 casos solamente en Varzea Grande. El origen de los surtos puede ser fácilmente asociada a la mala utilización de la estructura urbana, o sea, la cantidad de áreas abandonadas adentro del perímetro urbano que, de acuerdo con registros de la Secretaría de Infraestrutura (SINFRA), suman 68 mil terrenos baldíos, siendo que de éstos, 34 mil presentan malas condiciones (mato alto, sirven de depósito irregular de basura y facilitan la proliferación del mosquito transmisor de dengue).

A la primera vista los problemas citados, aun cuando de gran importe para el municipio, parecen inconexos sin embargo, al reflejar sobre tales problemáticas, percibiese una posible medida paliativa, en una actividad un tanto cuanto olvidada por el poder público local: la agricultura urbana. Autores como Mougeot (2000) y Dias (2000) apuntan en sus respectivos estudios que la agricultura urbana y periurbana consiste en una forma de suplir las ciudades con alimentos, proporcionando seguridad alimentaria, nutricional y haciendo mejor usos de los espacios urbanos otrora abandonados. En Mato Grosso se registra el ejemplo del municipio de Sinop, donde existe la agricultura urbana en el modelo de huertas escolares, que proporciona reducción de los dispendios con comida escolar, bien como, contribuye para la revitalización urbana y salud pública.

A idea de que la agricultura urbana promueve la seguridad alimentaria y auxilia en el control de enfermedades, en resultado del manejo adecuado de áreas en el perímetro urbano, es reforzada por Machado y Machado (2002) y Ribeiro (2012), al apuntar que la limpieza necesaria para el mantenimiento del plantío en áreas urbanas y periurbanas apoqué la proliferación de roedores e insectos y, por consecuencias, posibles enfermedades causadas por ellos. En este contexto, teniendo en vista la problemática presentada, ¿cuál sería la importancia de la horticultura para el municipio de Varzea Grande y cuál la capacidad de combatir las problemáticas de seguridad alimentaria y salude pública locales? Para aclarar estos questiomentos, como objetivo general se proponen identificar y caracterizar la agricultura urbana y periurbana (horticultura) en Varzea Grande en el año de 2013. Específicamente, en el primer momento, buscase localizar a los puntos donde la actividad es desarrollada en el perímetro urbano y periurbano y, posteriormente, analizar su dinámica y su estructura productiva.

En el campo académico, el trabajo tiene su justificativa debido a la falta de estudios del género en la región, de forma a traer una contribución inédita, tratada no solo por el lado social, pero también por el económico. Aún, la propuesta de iniciativas de investigación local con designio de promover transformaciones benéficas para una determinada comunidad no es nueva; tal colocación ya afuera expresa por diversos pesquisidores, entre ellos Navolar et al. (2010) y Ribeiro (2012), a lo afirmen que el 
envolvimiento de las personas con la producción alimentar en ambientes urbanos puede impactar positivamente la salud humana al introducir calidad de vida a los ambientes.

Para evaluación y discusión de los datos logrados, además de la caracterización de la producción local, fue utilizado el modelo de Análisis de Agrupación (jerárquico, aglomerativo y por encadenamiento medio), también conocida como Análisis de Clusters, método que permite clasificar las experiencias encontradas de grupos según a semejanza, de forma a facilitar la observación de calidades de comportamiento y características predominantes en la población estudiada. La estructura es compuesta por más tres secciones además de esta primera introductiva, con una discusión teorético-metodológica, seguida por la discusión de los resultados y de las consideraciones finales.

\section{ASPECTOS TEORÉTICOS Y METODOLOGICOS}

Un de los primeros conceptos de agricultura urbana y periurbana afuera presentado por la Organización de las Naciones Unidas para Alimentación y Agricultura (FAO) en 1999, definiéndola como pequeñas áreas ubicadas adentro del perímetro urbano, destinadas a la producción de cultivos de productos agrícolas e/o creación de pequeños animales para consumo propio e/o para comercio. En cuanto a la agricultura periurbana, se definió áreas de producción en las periferias de las ciudades, que podrían ejercer intensivamente las actividades, siendo ésas con designio comercial o no. La crítica en cuanto a esta definición vendría a sobre la subjetividad del término periferia, o a lo cual su entendimiento variar.

Losada et al. (1998) y Moustier (1998) trabajan el concepto que búsqueda definir las fronteras de urbano y periurbano, en una relación entre centro urbano y la distancia máxima que ése puede abastecer diariamente. Ya Losada (1998) buscó trabajar los espacios en tres categorías: las zonas urbanas, arrabaleras y periurbanas, donde el factor de delimitación daba en acuerdo con la cantidad de construcciones de edificios, infraestrutura y espacios no utilizados por kilómetros.

En la tentativa de suprimir las lagunas que circundan el concepto de agricultura urbana, algunos autores buscaron tipografar o clasificar la agricultura urbana y periurbana caracterizando el objeto de estudio. Mougeot (2000) conceptuó agricultura urbana según la actividad económica en que la producción y la venda deberían estar relacionados en el tiempo y en el espacio urbano debido a que mayor proximidad y al flujo rápido de recursos. Agricultura urbana y periurbana son entendidas cómo la actividad agrícola practicada adentro o en la periferia de los centros urbanos, siendo cultivadas, producidas, o criadas con variados productos alimenticios o no (flores), que serán ofertados en la misma área urbana o cercanías de dónde están ubicados. Además de la conceptualización basada en la actividad económica y su relación con tiempo y espacio, Mougeot (2000) consideraba el tipo de la relación del productor con la tierra, tanto por aspectos ocupacionales (si el productor residía en el área trabajada o no), cuanto por aspectos oficiales del uso del solo, como áreas habitacionales, industriales o institucionales.

A FAO (1999) también lanzara mano de clasificación para facilitar el estudio de la agricultura urbana y periurbana, con la clasificación de los sistemas de producción. Destaca a acuicultura (creación de pescado y mariscos), a horticultura (plantío de hortalizas, flores, frutos, legumbres y hierbas medicinales), a 
agrofloresta (plantío frutas, producción de semillas y compuestos), las creaciones (animales y derivados) y diverso (plantas exóticas, etc.).

Otros autores buscan clasificar la agricultura urbana en acuerdo con el objetivo de estas, como es el caso de Lopes (2004), que hay centrado en la Horticultura y clasifica los tipos de huertas en escolares, comunitarias y terapéuticas. Según el referido autor, huertas escolares o institucionales son, en general, trabajadas por la comunidad escolar, o sea, son cultivadas por los alumnos, padres y maestros. Posee diversos objetivos que varian entre la seguridad alimentaria hasta el auxilio al proceso de aprendizaje.

Del punto de vista de la seguridad alimentaria, las huertas refuerzan a comida escolar con el suministro de productos de calidad y confiabilidad, estimulando una practica de alimentación saludable, por medio del consumo de hortalizas. Como herramienta de aprendizaje, puede auxiliar en la enseñanza de fundamentos de biología, ecología y, en escuelas tecnológicas, en la reproducción y creación de técnicas de manejo. Tales huertas aún presentan la posibilidad de incentivar la reducción de basura, o sea, los residuos orgánicos producidos en esas escuelas pueden ser utilizados como adobo, además de la posibilidad de la reutilización de embalaje para mudas. Hay también el factor recreativo, una vez que las actividades agrícolas pueden ser presentadas como actividades extraescolares, con enfoque de trabajo cooperativo, o mismo como una alternativa de ocio.

De acuerdo con Lopes (2004), la denominación de huertas comunitarias corresponde a la otra clasificación importante a ser destacada (común en Rusia), por incorporar la participación de la comunidad, sin embargo, en la mayoría de los casos, administrada por el poder público. Huertas comunitarias están localizadas principalmente en áreas urbanas desocupadas, públicas o mismo particulares y la producción principal gira en torno a hortalizas, plantas medicinales, legumbres, frutas, otros alimentos y hasta mudas. Actualmente este tipo de huerta puede destinar su producción para restaurantes comunitarios que venden comidas a bajos precios, factor que fortalece la contribución de la agricultura urbana para con la seguridad alimentaria.

La agricultura urbana y periurbana, por medio de huertas comunitarias, también constituye una forma de utilización óptima de espacios urbanos, pues auxilia en el combate a I acumulación de basura, además del crecimiento de matorrales en el ambiente urbano. Tales espacios, cuando en malo estado de conservación, abrigan insectos venenosos y pequeños animales prejudiciales a la salud humana; así, la presencia de huertas en el ambiente urbano impactaría en la reducción de enfermedades transmitidas consecuentes de estos factores. Agregado a eso, las huertas urbanas mejoran el escurrimiento de aguas de las lluvias y, por consecuencia, auxilian en la mitigación de locales propicios a la reproducción del mosquito transmisor del dengue, un de los problemas substanciales relacionado al municipio estudiado.

Hay también la cuestión de la posibilidad de complemento de renta, teniendo en vista que muchas de esas experiencias poseen también el designio comercial. Las huertas comerciales buscan las ganancias y pueden presentar algunas características distintas: i) especializada, aquéllas en las cuales son plantadas a lo más tres variedades de plantas; ii) diversificada, donde hay plantío de más de tres especies de plantas; iii) educativas, con designio de enseñar técnicas de plantío (generalmente vinculadas a escuelas técnicas y 
órganos de soporte y regulación de la actividad agrícola); iv) experimentales, que sirven para tests con noticias técnicas de plantío y nuevos cultivar y $v$ ) caseras, con designio exclusivo de complementar la alimentación familiar, sin fines lucrativos, con poca o ninguna utilización de pesticida (LOPES, 2004).

En resultado de las consideraciones expuestas, el fortalecimiento de estudios sobre la agricultura en medio urbano tiende a fortalecer la actividad y su contribución en la producción de alimentos, una vez que no solamente potencialidades serán relacionadas, otrosí, habrá la posibilidad de identificar limitaciones que necesitan ser combatidas. En este caso, se considera el concepto de agricultura urbana y periurbana propuesto por Mougeot (2000), en que la dinámica de la actividad económica, la producción y la venda deben estar relacionadas en el tiempo y en el espacio urbano, a la mayor proximidad y al flujo rápido de recursos. Por medio de datos logrados junto a Secretaría de Estado del Medio Ambiente de Varzea Grande (SEMA/VG) afuera posible identificar que, geográficamente, la demarcación de Porto Velho no es considerada rural por el poder público municipal y también afuera considerada área de pesquisa, en acuerdo con la Figura 1.

En la Figura 1 está destacado el perímetro urbano del municipio de Varzea Grande $\left(132,92 \mathrm{Km}^{2}\right)$ y la región periurbana correspondiente a la demarcación de Porto Velho $\left(17,22 \mathrm{Km}^{2}\right)$ los márgenes de Rio Cuiabá. Según la SEMA/VG, la demarcación de Porto Velho no presenta fortes características rurales debido a que su proximidad de la capital mato-grossense, sin embargo, dispone de infraestrutura superior a las zonas rurales. Datos referentes a la localización de actividades agrícolas presentes en la región urbana y periurbana de Varzea Grande fueron logrados junto a SEMA/VG y por medio de fotos por satélite originados en el municipio.

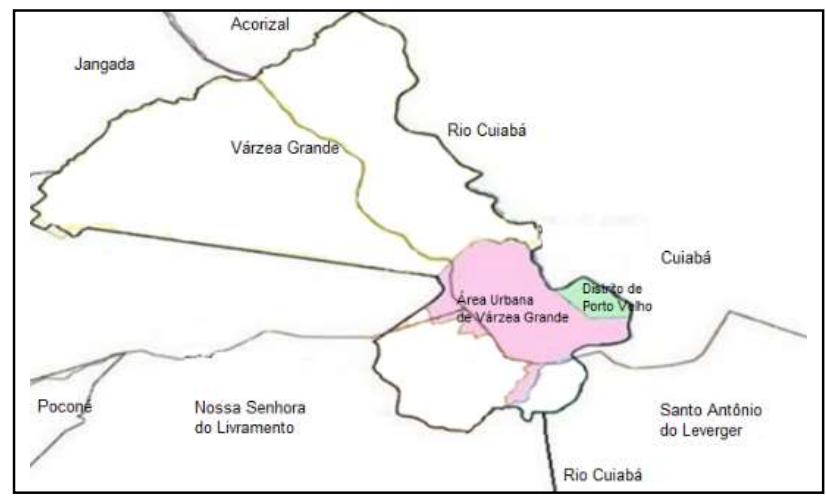

Figura 1: Mapa de Varzea Grande: delimitación del área abarcada en 2013. Fuente: IBGE (2012).

Basado en el trabajo de Ribeiro (2013), un cuestionario semiabierto fue montado de forma que, en la primera parte, contemplase cuestiones que visan identificar las características sociales de los productores, como edad, nivel de escolaridad, constitución familiar, participación de cónyuge e hijos en la obtención de la renta, origen de la renta, entre otras cuestiones con la misma finalidad. La segunda parte del cuestionario buscó captar informaciones que definen la dinámica productiva, el volumen producido, o sea, identificar lo que era producido, cuanto era producido y como era producido. En ese sentido, se tienen cuestiones referentes los tipos de hortalizas cultivadas, la cantidad y área plantada, tipo de fertilización e irrigación, allende cuestiones referentes a la seguridad, infraestrutura y comercialización.

Estos datos, allende posibilitar una evaluación descriptivo-qantitativa de la actividad, permitió que fuese realizada el Análisis de Cluster (jerárquico, aglomerativo y por encadenamiento medio). El análisis de 
agrupación auxilió en la clasificación de las experiencias encontradas de grupos en virtud de la semejanza entre los mismos, de forma a auxiliar en la observación de padrones de comportamiento y características predominantes de la población estudiada.

De acuerdo con Regazzi (2001), el Análisis de Clusters se trata de una metodología numérica multivariada, que tiene por objetivo proponer una estructura clasificatoria de grupos según algún criterio de homogeneidad, o sea, encontrar y despegar objetos en grupos similares. Al estudiar todo un conjunto de relaciones interdependientes, el Análisis de Agrupaciones no hace distinción entre variables dependientes e independientes, de tal modo que no presenta una relación causa efecto como en una regresión.

Malhotra (2001) apunta que el Análisis de Agrupaciones es un método simple, basado en cálculos de distancia, no siendo necesario conocimiento estadístico avanzado para aplicarlo, sin embargo, son necesarios cuatro conceptos: i) esquema de aglomeración, que informa sobre objetos a ser acordados en cada cursillo de un proceso jerárquico de aglomeración; ii) centróide de agrupación, que se trata de los valores medios de las variables para todos los objetos del análisis; iii) centros de agrupaciones, utilizados en agrupación no jerárquica, puntos iniciales cuyo entorno los grupos son formados, y iv) concepto de composición de agrupación, que presenta cual grupo cada objeto Pertence. Adentro del Análisis de Agrupaciones existen modelos jerárquicos y no jerárquicos, como puede ser observado en la Figura 2.

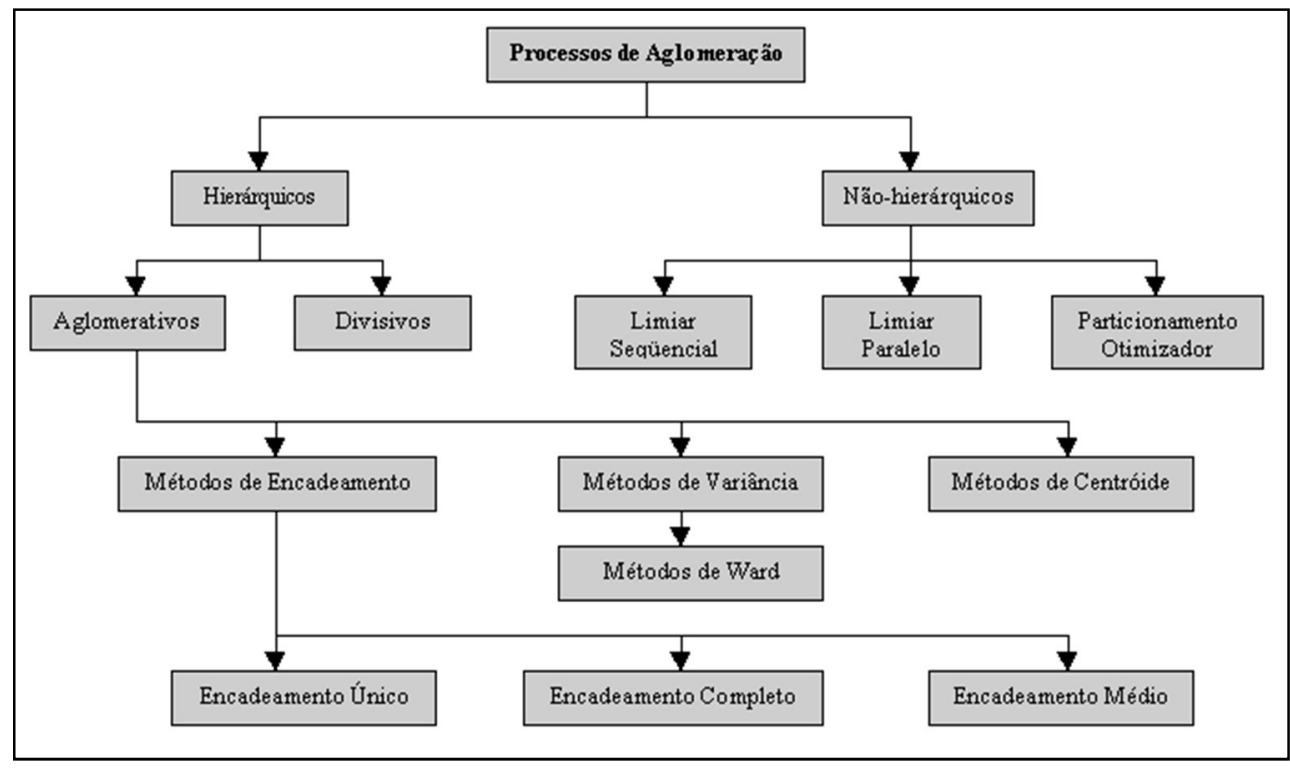

Figura 2: Procesos de Aglomeración. Fuente: Malhotra (2001).

Entre los modos de aglomeración, se destacan los jerárquicos y no jerárquicos; este último, también conocido como k-means (k-medias) trabajan con una predeterminación de la cantidad de grupos a ser formados, caracterizándose por la formación de grupos homogéneos, sin considerar la jerarquía entre los grupos. Los jerárquicos, subdivididos en aglomerativos y divisivos, establecen una orden jerárquica representada en una forma de árbol. Adentro del Análisis de Agrupaciones son más comunes los estudios que utilizan proceso jerárquico, subdivididos en método de encadenamiento, métodos de yerros de sumas de cuadrados, o métodos de variancia y métodos centroides (VICINI, 2005). Por su vez, los métodos divisibles, trabajan desde un único grupo inicial con todos los objetos y éstos son subdivididos en subgrupos de máxima 
semejanza entre ellos, ésos son novamente subdivididos a la medida que la semejanza entre los grupos vaya apocando hasta queden tantos grupos cuanto observaciones (GIGANTE et al., 2007).

El Análisis de Agrupación a través del método jerárquico presenta una representación gráfica de sus resultados conocido por los nombres de Dendograma, Fenograma, o mismo, gráfico en árbol. Por si tratar de una síntesis de los resultados el Dendograma presenta una pequeña pérdida de información, sin embargo, tal pérdida y considerada irrelevante, una vez que la herramienta posee gran utilidad para comparación y clasificación de grupos.

Cuando a los modos de representación gráfica, el Dendograma puede ser elaborado de forma vertical u horizontal. En un Dendograma horizontal el eje y representa los grupos unidos por orden decrecientes mientras el eje $\mathrm{x}$ presenta la distancia entre los grupos formados. En un Dendograma horizontal la lectura interpretativa debe ser hecha de arriba para bajo; se observa en la Figura 3 que el grupo (A, B) es lo que presenta menor distancia y, por tanto, mayor semejanza adentro del Dendograma, en consecuencia, tiene si el grupo (C, D) demostrando que los grupos son presentados en orden decreciente de semejanza.

Lo según modo de presentación es el vertical, en que el eje y representa la distancia entre los grupos formados y el eje $\mathrm{x}$ representa los grupos formados por orden decreciente de semejanza, siendo su lectura efectuada de la derecha para la izquierda. Escogido el proceso de aglomeración considerado adecuado, se pasa la selección de los parámetros de distancia, que según Regazzi (2001) es tenido como un problema común en el Análisis de Agrupaciones, dado que con el cambio entre medidas se puede lograr resultados diferentes. De tal forma, es necesario que se test las diferentes medidas, en el designio de encontrar resultados gráficos con menores distorsiones de la realidad estudiada.

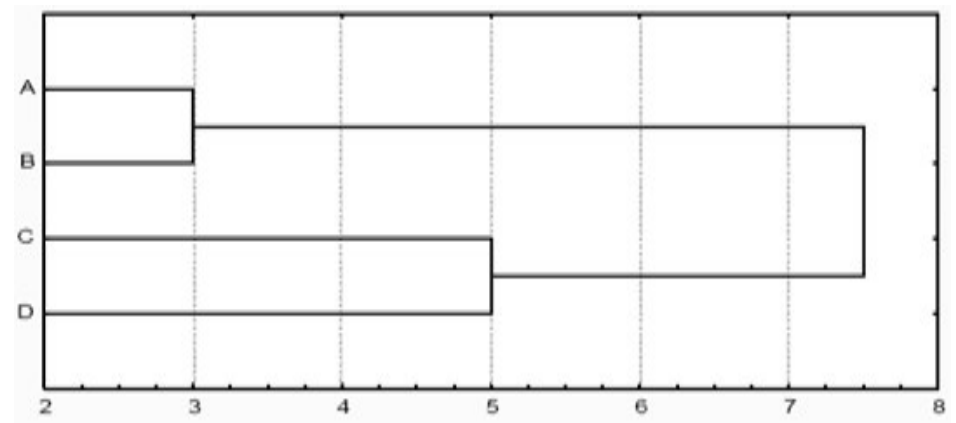

Figura 3: Dendograma Horizontal. Fuente: GeoCities (2012).

Entre los parámetros de distancia, o coeficientes de dissimilaridad, lo más comumente utilizado es la distancia euclidiana, en la cual la distancia entre grupos es dada mediante el teorema de Pitágoras en un espacio multidimensional, un punto negativo de la distancia euclidiana es que ésa puede presentar graves distorsiones suponiendo no haya la estandarización de los datos de unidades diferentes. Aún existe la distancia euclidiana media donde la distancia entre dos grupos es encontrada por la media de las distancias y la distancia de Mahalanobis, que es utilizada como medida cuando existe repetición de datos entre dos poblaciones que son igualmente de común utilización (VICINI, 2005).

Dada naturaleza del banco de datos y los objetivos propuestos, el análisis utilizado afuera jerárquica, aglomerativa y por encadenamiento completo, sin embargo, se abre espacio para presentar las diferencias 
entre los tipos de encadenamiento. El encadenamiento único (Single Linkage), o vecino más prójimo (Nearest Neighbor) es el proceso en el cual primeros objetos agrupados son los que presentan menor distancia entre sí. En la secuencia se encuentra menor distancia entre un objeto y el grupo recién formado, componiendo un tercero y así sucesivamente. En cada etapa la distancia entre dos grupos será igual a la distancia de sus variables más prójimas. El proceso continúa hasta si formar un único grupo.

En la Figura 4 se observa que la distancia de los grupos más prójimos es medida por la distancia de las variables más prójimas. Autores como Valentin (2000) y Vicini (2005) apuntan que, debido a que su llaneza, el método posee una desventaja, o sea, al reunir el elemento más prójimo a los grupos reciénformados, los objetos estraperlistas serían rápidamente aglomerados, resultando en una dificultad en la identificación de cada grupo.

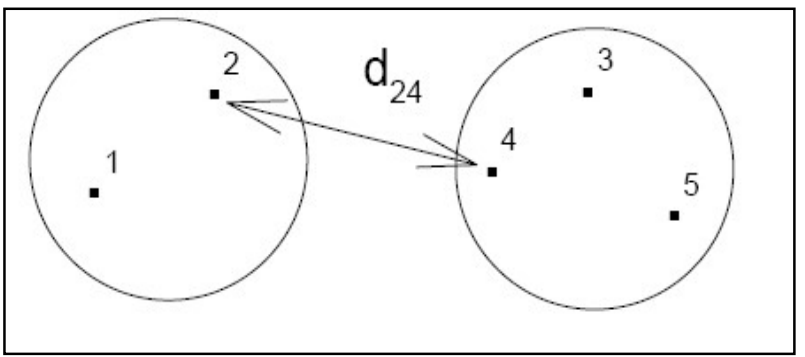

Figura 4: Encadeamento Único. Fuente: Gigante et al. (2007).

El encadenamiento completo (Complete Linkage) o vecino más distante (Farthest Neighbor) consiste en el cálculo de la distancia de los objetos más prójimos, considerando sus variables más distantes, conforme Figura 5. En detrimento al encadenamiento simple, en el completo se puede afirmar que un objeto solamente irá a si agregar a un grupo si las demás variables son más prójimas que la del par en cuestión. De tal modo, cabe resaltar que los grupos aún son formados con objetos de mayor semejanza, sin embargo, su distancia es calculada con base en sus variables más distantes. A asignación de los objetos, en primera estancia y en matrices de datos relativamente pequeños tienden a no diferir; lo que alteraría entre el encadenamiento simple y lo completo sería la distancia de los grupos formados.

La ligazón media o encadenamiento medio (Average Linkage), método utilizado en este estudio, semejante a los demás procesos de encadenamiento, se basa en la formación de grupos de objetos más prójimos, en la distancia media entre todos los pares de variables. En este método no son considerados apenas la distancia mínima o máxima, pero las distancias de las variables como un todo, como puede ser observado en la Figura 6.

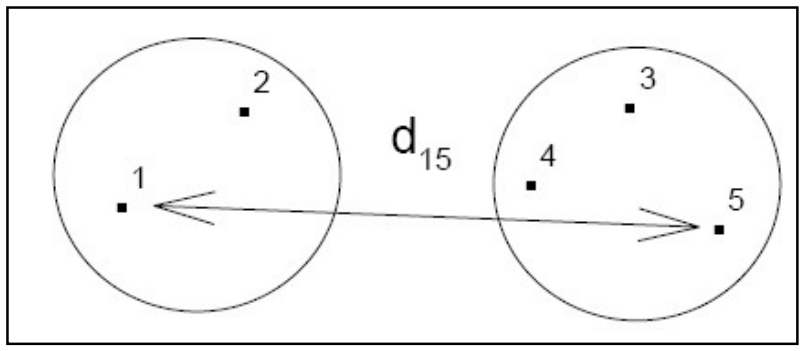

Figura 5: Encadeamento Completo. Fue Fuente: Gigante et al. (2007). 


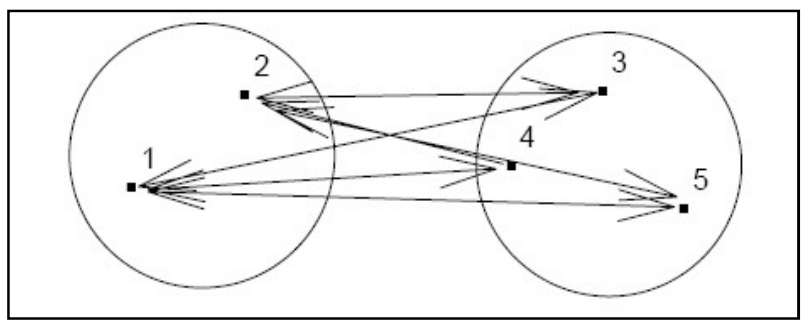

Figura 6: Encadeamento Medio. Fuente: Gigante et al. (2007).

Se considera que el Análisis de Agrupaciones, en el contexto de este estudio, apunta su aplicación para la mejor caracterización de las experiencias de agricultura urbana y periurbana en Varzea Grande, de modo a identificar la semejanza entre objetos o casos por medio de algoritmos y consistencia estadística. La aglomeración jerárquica, aglomerativa y por encadenamiento medio afuera utilizada para aspectos económicos y sociales en el proceso de caracterización del objeto de estudio, de modo la mejor identificar experiencias con características semejantes.

\section{RESULTADOS E DISCUSIONES}

Desde la investigación realizada, se encontró un número reducido de experiencias que se encajaban en el parámetro de estudio propuesto (horticultura ejercida en el perímetro urbano y periurbano aparte de las demarcaciones rurales). Fueron identificadas 19 observaciones, siendo 9 ubicadas adentro del perímetro urbano y 10 en el periurbano. En el perímetro urbano las actividades encuentran si dispersas mientras en el periurbano las observaciones se disponen a lo largo de la segunda mitad de la Alameda Júlio Müller, en la Avenida Ribeirão y en la Carretera del Engordador, todas a los márgenes del Rio Cuiabá. La representación gráfica de los casos apuntados están expuestos en las Figuras 7, 8, 9 y 10.

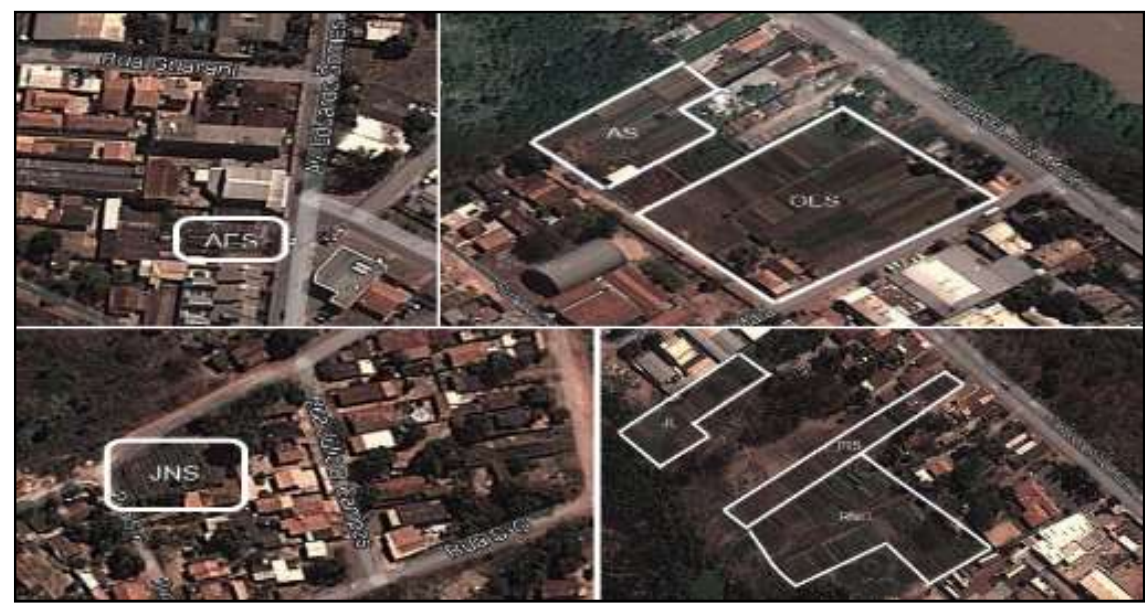

Figura 7: Experiencias de Agricultura Urbana em Varzea Grande, 2013. Fuente: Google Mapas (2013).

En la Figura 7 es posible identificar la disposición de nueve casos de agricultura urbana, LAS sitúa si prójimo el ayuntamiento municipal de Varzea Grande en la villa Ipase, JNS está en el barrio Jardín Gloria I, prójimo la carretera de la garita que es vía para Puente Sérgio Motta, las experiencias LAS, LOS, JL, IRS y RNO están ubicadas a lo largo del primer trecho de la Alameda Júlio Müller, prójimo a el Rio Cuiabá y prójimo el Puente Júlio Müller. Las actividades LAS y JNS poseen área de plantío reducido cuando comparadas las demás, tal característica es debido a la gran inserción en el núcleo urbano. 
La Figura 8 representa tanto experiencias de horticultura encontradas en el perímetro urbano como en el periurbano. En la Figura 8 están representados casos de agricultura urbana y periurbana: MA se encuentra a lo largo de la Carretera del Engordador prójimo el Rio Cuiabá; las experiencias RC y MF están localizadas en el barrio Unipark, a pesar de estén ubicadas prójimas a la demarcación de Porto Velho, aún encuentranse en el perímetro urbano.

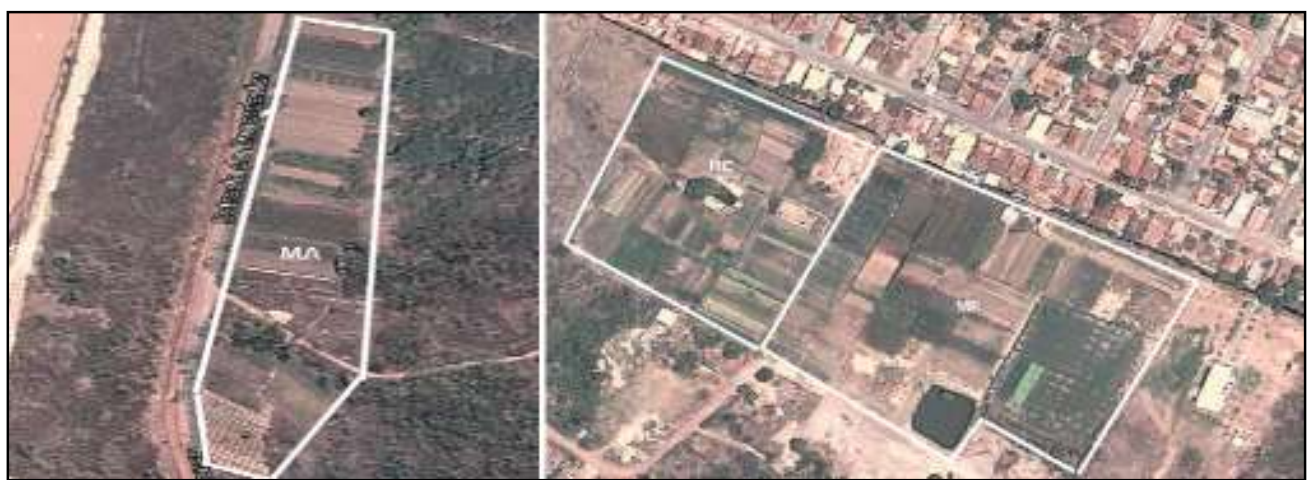

Figura 8: Experiencias de Agricultura Urbana y Periurbana en Varzea Grande, 2013. Fuente: Google Mapas (2013).

La Figura 9 presentan apenas los casos periurbanos encontrados, específicamente los situados en la segunda parte de la Alameda Júlio Müller, adentro de la demarcación de Porto Velho, y en la Avenida Ribeirão. En la Figura 9 están representados cuatro casos, entre ésos apenas JO no trate si de un área arrendada. La experiencia JPQ desarrolla si una región de poca visibilidad; en entrevista con el responsable por la actividad, ése alegó que la plantación será descontinuada y posteriormente retomada en el terreno murado prójimo a CES.

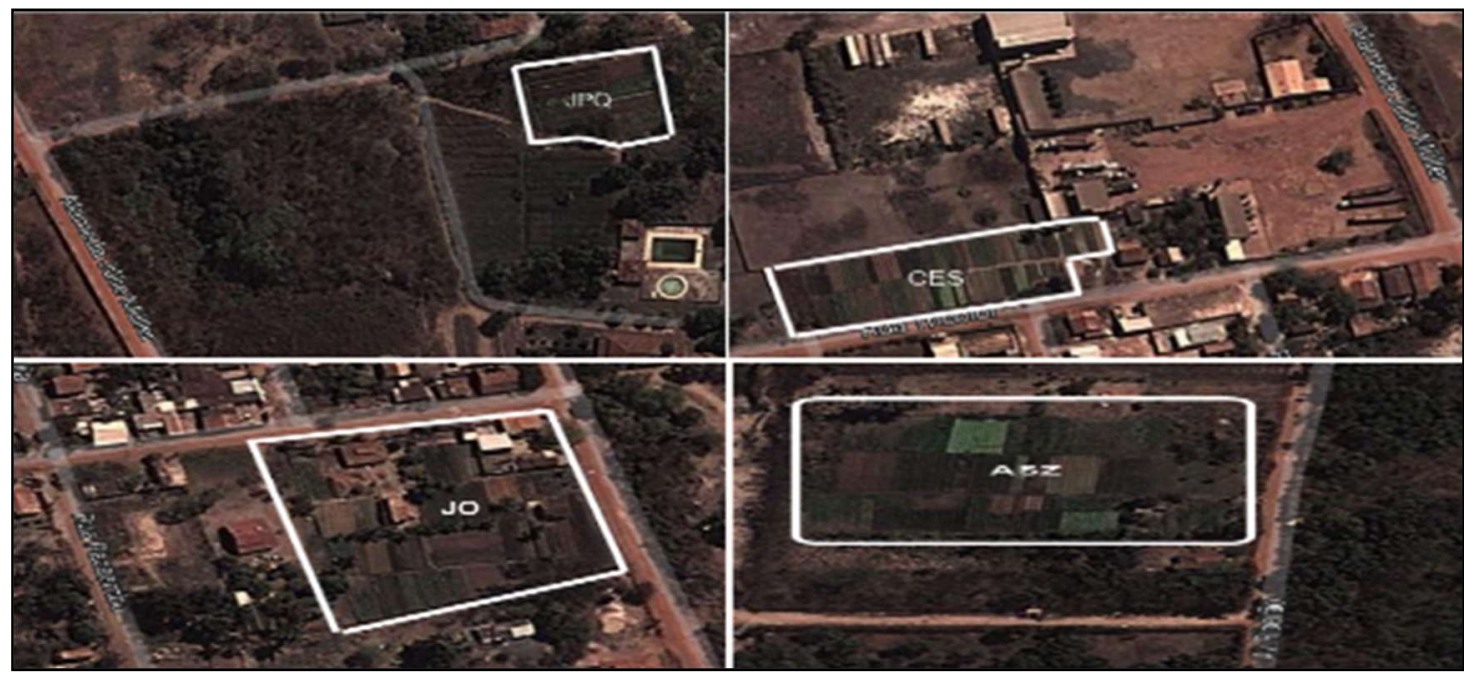

Figura 9: Experiencias de Agricultura Urbana y Periurbana en Varzea Grande, 2013. Fuente: Google Mapas (2013).

En la Figura 10 están presentes cinco casos de horticultura, todos los terrenos son arrendados, siendo que los terrenos donde las actividades VV y AL son practicadas poseen el mismo propietario. Las experiencias $\mathrm{NT}, \mathrm{HE}, \mathrm{VV}$ y Al están separadas por pocos metros, sin embargo, hay poca o ninguna interacción entre los agentes envueltos. 


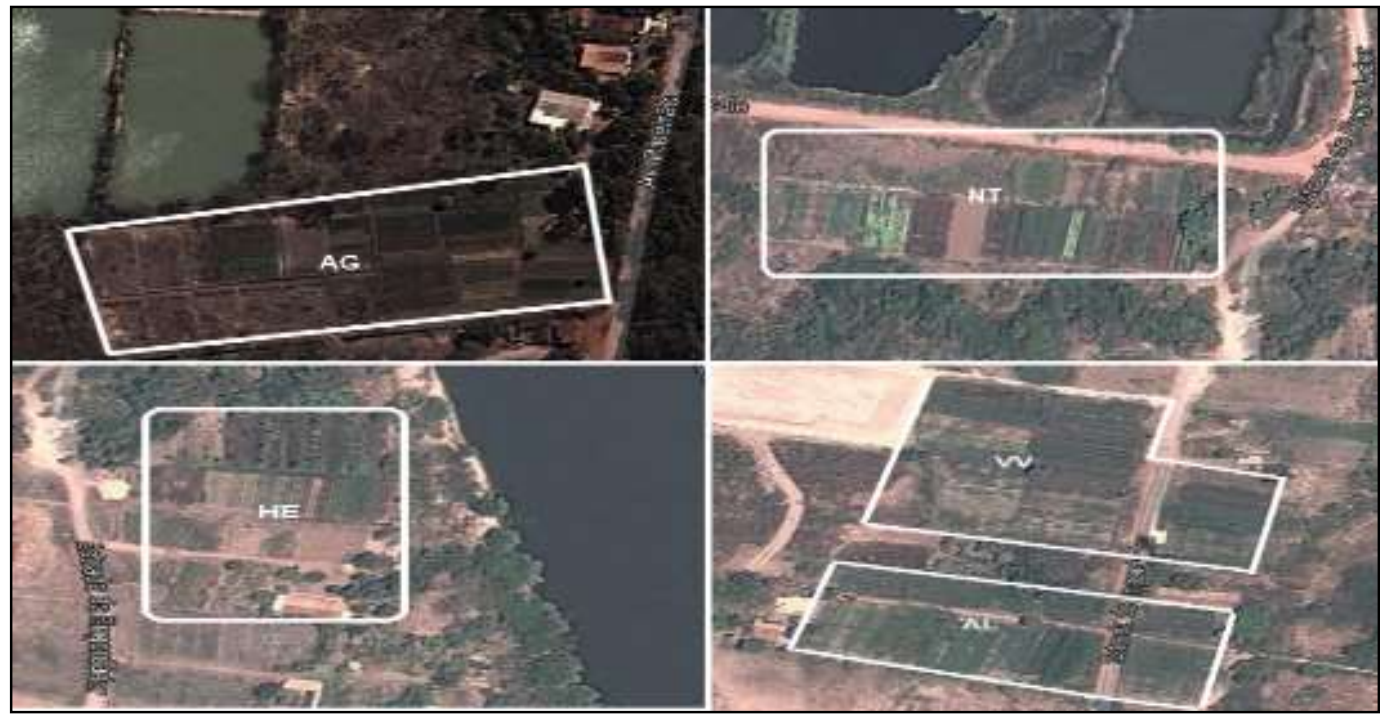

Figura 10: Otras Experiencias de Agricultura Periurbana de Varzea Grande, 2013. Fuente: Google Mapas (2013)

Las tres experiencias en las cuales a horticultura representa un percentual inferior a el $75 \%$ de la renta mensual sano a JNS (Figura 13), RNO y HE. JNS tiene su principal fuente de renta en la construcción civil, mientras RNO y HE son jubilados que buscaron complementar la renta por medio de la horticultura. Las tres experiencias poseen en común la función primaria de completar la alimentación de la familia.

Los casos estudiados fueron agrupados en acuerdo con la semejanza de sus características sociales, los resultados están explícitos en el Cuadro 01 y en la Figura 11. En la Figura 11 está representado un Dendograma de semejanza según características sociales. El Grupo 01 formado por LAS es menos similar a los debido a que poseer características que discuerdan mucho del contexto general; LAS es el único productor que presenta un nivel elevado de escolaridad y el Grupo 02 compuesto por JNS, RNO y HE presentan semejanza entre sí, sin embargo, discuerdan de los demás una vez que es compuesto por aquellas experiencias que no son realizadas exclusivamente para obtención de ganancias.

El Grupo 03 es compuesto por los productores en que la actividad puede ser considerada tradición familiar y a horticultura es principal fuente de renta. Mismo a pesar de han heredado las técnicas de plantío, están abiertos al aprendizaje de nuevas técnicas y complementan la renta con actividades no agrícolas. El Grupo 04 tiene componentes que no presentan apertura para aprendizaje de noticias técnicas de manejo y diferentemente del Grupo 03 no ejercen actividades que complementen la renta fuera de la agricultura, sin embargo, también poseen fuerte influencia de la cultura local. El Grupo 05 presenta los casos con características más similares; los productores de este grupo presentan faja etaria entre 29 a 45 años, poseen su principal fuente de renta en la horticultura y actúan solamente con productos agrícolas y su comercialización. Sin limitaciones culturales, a pesar del bajo nivel escolar, desean aprender técnicas que mejoren la productividad. Dadas a las características encontradas y representadas en el Dendograma, se puede afirmar que de modo general los horticultores de la región urbana y periurbana de Varzea Grande poseen un bajo nivel de instrucción académico y técnico, poco si distinguiendo unos de los otros. Hay expresiva semejanza en cuanto a medios de obtención de renta, dado que once de las diecinueve 
experiencias logran renta exclusivamente de actividades agrícolas. Cabe resaltar que, en cuanto la rentabilidad, apenas afuera posible alzar datos referentes la renta bruta semanal.

Una de las justificativas de la realización de este estudio es percepción de la agricultura urbana y periurbana como modo de mejor aprovechamiento de espacios otrora abandonados, contribuyendo indirectamente para la salud pública. Buscando verificar tal preposición en las experiencias encontradas, se alzo datos referentes a variables que pudiesen favorecer o nivelar los beneficios de un terreno limpio y bien cuidado y, para tanto, se identificó como ocurre la utilización de pesticidas y cual tipo de agua era utilizada en la irrigación. Por medio de los datos logrados, las experiencias fueron agrupadas en acuerdo con la semejanza entre las mismas y los resultados pueden ser observados en el Cuadro 02 y en la Figura 12.

Cuadro 01: Grupos de Horticultores Según las Semejanzas de sus Características Sociales, 2013.

\begin{tabular}{|c|c|c|c|c|}
\hline GRUPO 01 & GRUPO 02 & GRUPO 03 & GRUPO 04 & GRUPO 05 \\
\hline AES & JNS & AG & JL & IRS \\
\hline & RNO & AS & VV & OES \\
\hline & HE & CES & RC & ASZ \\
\hline & & & NT & AL \\
\hline & & & JPQ & MA \\
\hline & & & & MF \\
\hline
\end{tabular}

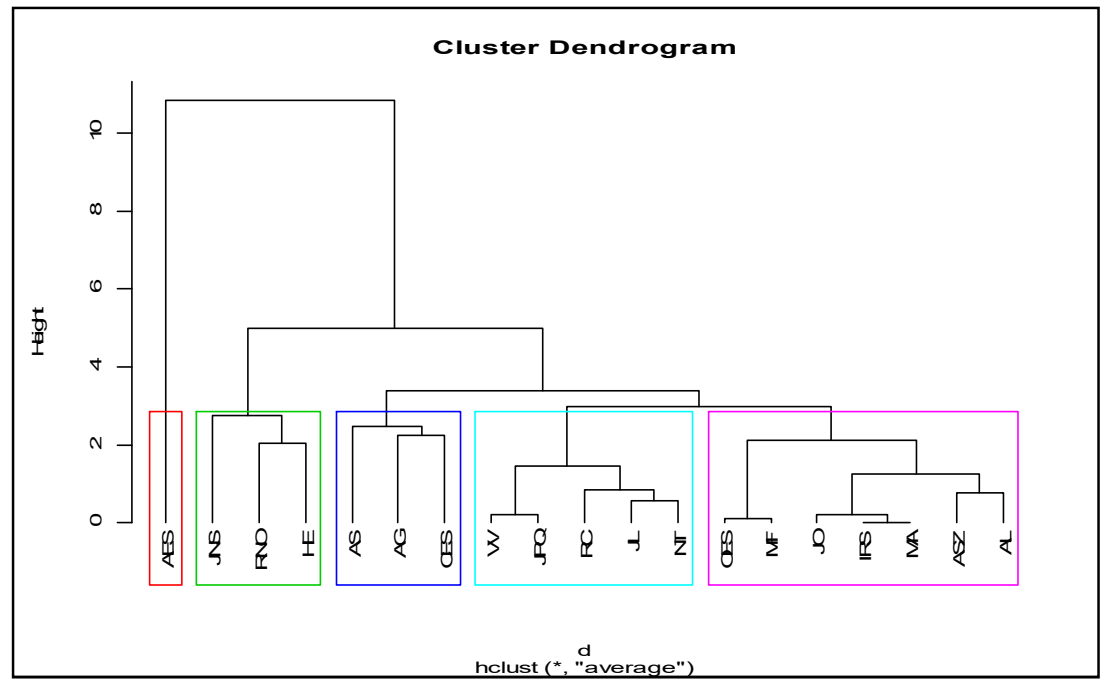

Figura 11: Dendograma Referente las Características Sociales de los Horticultores Entrevistados, 2013.

Quadro 02: Grupos de Horticultores Según las Semejanzas de las Características Ambientales, 2013.

\begin{tabular}{|c|c|c|c|c|}
\hline GRUPO 1 & GRUPO 2 & GRUPO 3 & GRUPO 4 & GRUPO 5 \\
\hline AS & AES & JNS & MA & AG \\
\hline & IRS & JL & & JT \\
\hline & OES & HE & & \\
\hline RNO & RC & & \\
\hline VV & ASZ & & \\
\hline & AL & & & \\
\hline MF & & & \\
\hline
\end{tabular}

El Grupo 1, compuesto por LAS es lo más dissimilar, sin embargo, se trata de lo que menos posee impactos negativos sobre el ambiente y posiblemente sobre la salud de los potenciales consumidores. En el caso LAS, en que es producido preferencialmente el cilantro, el productor utiliza si de mariquitas (insectos 
predadores de la familia Coccinellidae del orden Coleoptera) en el combate de plagas, con la utilización de agua encañada que, por los parámetros de producción en el perímetro urbano y periurbano, puede ser considerado aceptable y sin grandes maleficios para el público consumidor.

Los Grupos 2 y 3 engloban experiencias donde agua utilizada no presenta impacto negativo perceptible para el terreno o consumidores, sin embargo, ambos utilizan de pesticidas para el combate de plagas; el Grupo 3 diferencia del 2 debido a los productores aleguen poseer entrenamiento para manejo de los pesticidas, allende poseer equipos de seguridad. El Grupo 4, compuesto por solamente el caso MA, también se utiliza de pesticidas y alega poseer entrenamiento para realizar el manejo, sin embargo, la señal de alerta o el factor que causaría preocupación cuanto posibles maleficios, sería a agua utilizada, una vez que algunos productores, incluyendo MA, no poseen preocupación con el origen del agua.

El Grupo 5 engloba los casos en los cuales los productores no poseen entrenamiento para el manejo de los pesticidas y utilizan agua de fuentes contaminadas, como lagos en la región del Engordador, repletas de cascotes y carcasas de animales, o de pozos artesianos mal planificados, o mismo del Rio Cuiabá por medio de bombas y sin tratamiento. Entre los diecinueve casos visitados, cuatro demostraron un escenario peligroso tanto para salud del productor, como de posibles compradores, anulando el efecto positivo indirecto para salud que un terreno manejado de forma correcta podría traer para la población.

El estudio buscó identificar como es realizada la comercialización de los productos agrícolas oriundos de las olericolas urbanas y periurbanas del municipio de Varzea Grande. Por medio de los datos levantados fue posible constatar que no existe cualquier tipo de contrato entre productores y vendedores; todas las transacciones son realizadas por contratos verbales y no existen padrones pre-establecidos para la cualidad del producto. A pesar de apenas el $32 \%$ de los casos estudiados no poseer compradores fijos (compradores que realizan adquisiciones con frecuencia al menos bisemanal en un período superior a un año), no hay estipulación previa de una cuantía fija, así la cantidad retirada es establecida en el momento que los compradores van a buscar el producto o requisan a entrega.

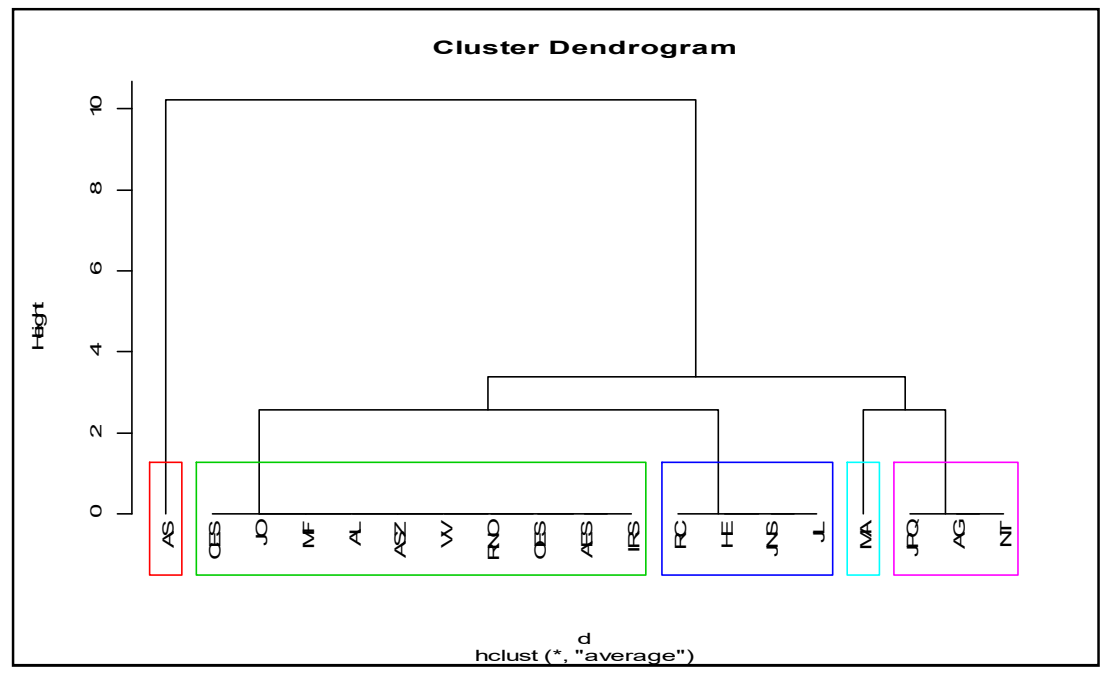

Figura 12: Dendograma Según Características Ambientales de los Horticultores Entrevistados, 2013. 
Se percibe, por la observación de la actividad, que pocos productores están ciertos de la baja inserción de mercado que poseen. Existe una serie de factores que contribuyen para que mismo, quien produzcan una cuantía considerable, no consiga poner las hortalizas en el mercado, por falta de condición de infraestrutura local, falta de seguridad e/o falta de visibilidad.

Utilizando la técnica de análisis de agrupación jerárquica, por la distancia euclidiana y ligazón media, se llegó al Cuadro 3, conteniendo los grupos sus semejanzas en el ámbito comercial. En la Figura 13 es de fácil observación dos grupos que son mucho dissimilares de los demás: el Grupo 1 es formado apenas por MF, que posee un modelo de comercialización diferente de todos los demás, una vez que el plantío es vinculado a una tienda de verduras, que costea parte de los insumos y adquiere toda producción. El Grupo 2 VV es formado por la experiencia, cuya toda a venta es hecha por medio de terceros.

Quadro 03: Grupos de Horticultores Según las Semejanzas de las Características Comerciales, 2013.

\begin{tabular}{|c|c|c|c|c|}
\hline GRUPO 1 & GRUPO 2 & GRUPO 3 & GRUPO 4 & GRUPO 5 \\
\hline MF & VV & IRS & RNO & AES \\
\hline & & OES & HE & JNS \\
\hline & & & AG & JL \\
\hline & & & ASZ & AL \\
\hline & & & MA & JPQ \\
\hline & & & JO & \\
\hline
\end{tabular}

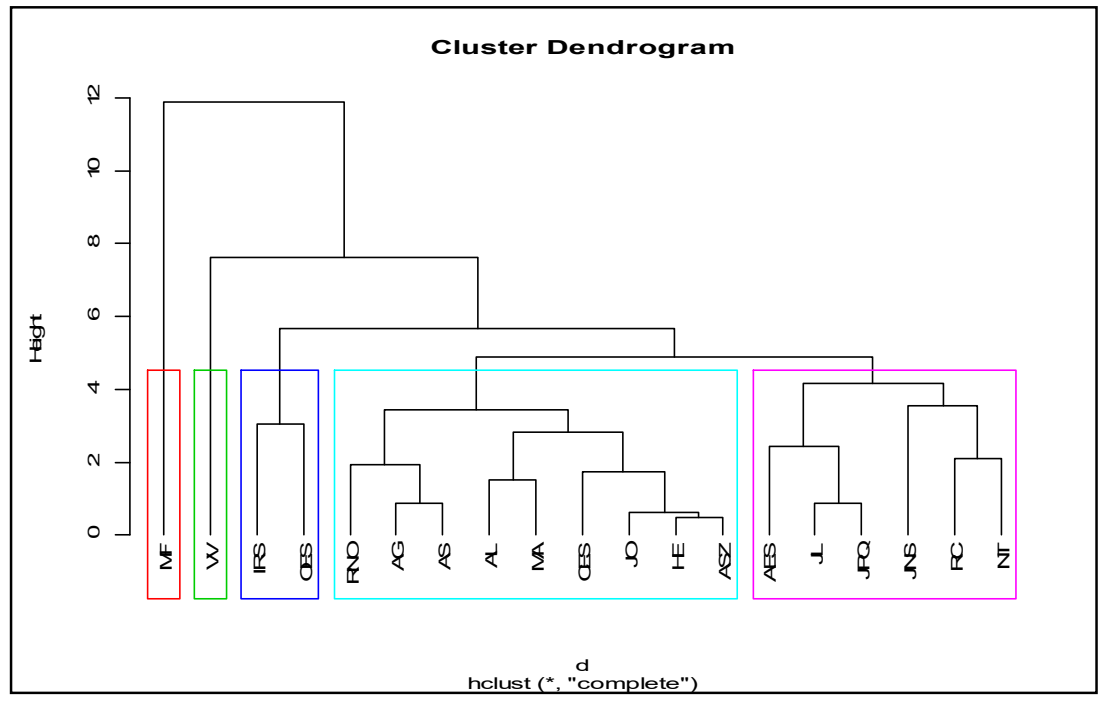

Figura 13: Dendograma Según Características Comerciales de los Horticultores Entrevistados, 2013.

El Grupo 3 engloba las experiencias IRS y LOS, que presentan semejanza en la cantidad de compradores fijos y en la destinación de los productos. El Grupo 4 es compuesto por productores que poseen menos de cinco compradores fijos, cuyas ventas son afectadas negativamente por falta de infraestrutura. El grupo 5 por su vez, es compuesto por las experiencias que poseen conciencia sobre a baja inserción en el mercado, pero atribuyen el hecho a un mercado consumidor pequeño. A pesar de la agrupación jerárquica formar grupos con mayor número de semejanza, en tenemos generales, no hay un caso que se destaque como excepcional en la cuestión de la comercialización, o sea, no hay un grupo en el cual sea evidente una forma adecuada de realizarse la comercialización. 
Del total de casos estudiados, el $29 \%$ distribuyen sus mercaderías en mercados y tiendas de verduras locales; ninguno productor entrevistado suministra para las grandes redes de supermercados del municipio. Entre los entrevistados, el 16\% alegaron suministrar para restaurantes en las cercanías, mismo porcentual de experiencias que suministran verduras para cocina de empresas con refectorios, que ofertan diariamente comidas a los empleados. Este tipo de venda ocurre diariamente, en pequeñas o medias cantidades.

La venta minorista in loco es realizada por el $58 \%$ de los casos, siendo la principal forma de comercialización. Los compradores son residentes prójimos las áreas de plantío y el flujo ocurre en unidades. La venta para feriantes es la tercera forma de comercialización y destinación más utilizada por los horticultores, englobando el $37 \%$ de los productores; en contrapartida, apenas el $26 \%$ de los productores posee tienda en ferias. Mismo produciendo en Varzea Grande, la totalidad de los horticultores que poseen tienda en ferias, apuntaron la comercialización para la capital Cuiabá, como la Feria del Verdão, la Feria del Porto y las ferias de barrio del Centro Político Administrativo. Tal situación ocurre debido al espacio insuficiente en la feria del centro de Varzea Grande, que solamente está abierta al público de viernes en el período nocturno hasta el domingo.

\section{CONSIDERACIONES FINALES}

La propuesta de este estudio tuvo como objetivo caracterizar la agricultura urbana y periurbana de Varzea Grande e identificar si la referida actividad aportaría en el combate a los problemas relacionados a la seguridad alimentaria y a la salud pública en el municipio, o mismo tratarse apenas de una actividad de complemento de renta. Algunas consideraciones pueden ser identificadas y, de la forma como la actividad se desarrolla actualmente, no puede ser sugerida para apocar los problemas apuntados.

Relativo a las cuestiones alimentarias del municipio, como en el caso de la falta de comida escolar, podería sí encontrar una medida paliativa en la adquisición de la producción de los horticultores urbanos y periurbanos, una vez que ésos no encuentran flujo para todo cuanto producen. Sin embargo, como prioridad sería necesario el entrenamiento de algunos productores para el manoseo correcto de pesticidas, además de la orientación y acompañamiento en cuanto la utilización de agua, buscando evitar la utilización de agua contaminada. Los productores ganarían al apocar la dependencia de terceros y el municipio ganaría en el precio pago por la producción. En cuanto la cuestión de la salud pública, afuera observado que en los terrenos donde las actividades son desarrolladas no hay acumulo de basura ni cascotes, de modo que precave la proliferación de animales e insectos nocivos. Todavía, como mencionado anteriormente, hay utilización inadecuada de pesticidas y de agua posiblemente contaminada, que puede generar nuevas situaciones de peligro para la salud pública.

El modelo actual de agricultura (horticultura) urbana y periurbana no se presenta como ventajoso, tanto en el aspecto de complemento de renta como en los de manutención de seguridad alimentaria y salude pública. Para tanto se hace necesaria a acción del poder público municipal, asociado las instituciones de investigación y enseñanza. El incentivo de la actividad, del modo como ocurre actualmente, generaría otras problemáticas de igual intensidad o superior. 
El primer paso para a mejora del cuadro sería concienciación del productor sobre sus métodos de plantío, al comprender qué la mayoría de las técnicas utilizadas pueden tornar la calidad del producto cuestionable y hasta mismo nocivo la salud. Lo según paso estaría en la construcción de una identidad de la clase productiva, sea a través del poder público o formando una entidad civil, con la percepción que los demás productores no serían únicamente competidores y formarse una fuerza representativa, que facilitaría el diálogo con el poder público e instituciones de incentivo a la producción agrícola.

Analizando el cuadro general de la actividad en el municipio de Varzea Grande, se comprende que pequeñas fuerzas productivas no consiguen "resistir" al "modelo del mercado" o mismo a acción prolongada de una política social desfavorable, lo que refleja en el deterioro de la actividad y de individuos dispuestos a comprometerse. Apenas el fortalecimiento por medio de acciones cooperativas podría proporcionar un comienzo de cambios en el cuadro. Tal perspectiva no es nueva o mismo innovadora, sin embargo, aún no hubiera esfuerzos por parte alguna para implantarla.

\section{REFERÊNCIAS}

DIAS, J. A. B.. Produção de Plantas Medicinais e Agricultura Urbana. Horticultura Brasileira, Brasília, v.18, 2000.

FAO. Food and Agriculture Organization. Cuestiones de la Agricultura Urbana. Revista Enfoques, 1999.

GIGANTE, L. A.; ZAVALA, A. A. Z.. Um Estudo da Similaridade das Queimadas entre Municípios no Estado de Mato Grosso. Revista Sinergia, v.11, n.2, 2007.

LOPES, F. J. R.. Apostila da Horta Comunitária. Campinas: GDR/CEASA. 2004.

LOSADA, H.. Urban Agriculturein the Metropolitan Zone of Mexico: changes over time in urban, sub-urban and periurban areas. Environmentand Urbanization, v.10, n.2. 1998.

MACHADO, A. T.; MACHADO. C. T. T.. Agricultura Urbana. Planaltina: Embrapa, 2002.

MALHOTRA, N. K.. Pesquisa de Marketing: uma orientação aplicada. Porto Alegre: Bookman. 2001.

MOUGEOT, L. J. A.. Agricultura Urbana: conceito e definição. In: BAKKER, N.. Cultivando Cidades, Cultivando Comida. Ottawa: IDRC, Canadá, 2000.
MOUSTIER, P.. La Complémentarité entre Agricultureurbaine et Agriculturerurale. In: SMITH, O. B.. Agriculture Urbaine em Afrique de I? Ouest: une contribution à lasécurité alimentaire et à l'assainissement desvilles. Wageningen: CTA/Ottawa: IDRC, 1998.

NAVOLAR, T. S.; RIGON, S. A.; PHILIPPI, J. M. S.. Diálogo Entre Agroecologia e Promoção da Saúde. Revista Brasileira de Promoção da Saúde, Fortaleza, 2010.

REGAZZI, A. J.. Análise Multivariada. Viçosa: UFV. 2001. RIBEIRO, S. M.. Agricultura Urbana Agroecológica Sob o Olhar da Promoção da Saúde: a experiência do projeto colhendo sustentabilidade. (Dissertação de Mestrado). São Paulo: USP. 2013.

RIBEIRO, S. M.. Agricultura Urbana Agroecológica: estratégia de promoção da saúde e segurança alimentar e nutricional. Fortaleza: RBPS. 2012.

SILVA, C. M.. A Produção Artesanal e Agricultura Familiar de Várzea Grande-MT. (Dissertação de Mestrado) Cuiabá: UFMT. 2010.

VICINI, L.. Análise Multivariada da Teoria à Prática. Santa Maria: UFSM. 2005. 